\title{
Study on Developing Green, Energy-saving, Long-acting and Water- washable Filter Product
}

\author{
Lin,Hsien-Ta \\ Department of Business Administration, TransWorld University \\ Email: sd471216@yahoo.com.tw
}

Keywords: Green Energy; Energy Saving; Water-Washable; Environmentally Friendly Filter

\begin{abstract}
This research focuses on the development of green, energy-saving, long-acting and water-washable filter and the non-woven fabric material of general filter to develop green products of long-acting and water-washable crude, primary and intermediate filter which can conform to the appeal of environmental protection and green energy; and then weaving technology, pleating technology and fiber fineness are used to improve product filtration efficiency, so that the product service life can last for 5 years and the long-acting and water-washable crude, primary and intermediate filter which can be washed by water for more than 60 times will be developed.
\end{abstract}

\section{Introduction}

This research takes water-washable, green and environmentally friendly filter as research subjects, in hope that the green products can break the original industrial rule via disruptive innovation and promote society to generate innovative service through such environmentally friendly product.

At present, filter material market is significantly potential due to the improvement of environmental protection and health concept; besides[1][2], there are other 8 reasons for it: the global demand for indoor artificial heating and cooling is increased, the migration of Asian population is frequent, the demand for sea water desalination is increased, food safety problem is increasing, the particulate matter in the air will become the preferentially controlled pollutant, the global fossil fuel is exhausted, ethyl alcohol will become the leading fuel in the future and the emission of greenhouse gases is reduced.

The polypropylene (PP) used in this research is provided by Taiwan Polypropylene[3][4]. The density of polypropylene fiber is $0.903 \mathrm{~g} / \mathrm{cm} 3$, and it is the lightest synthetic fiber among all the chemical fibers and also the only one which can float on the water. Due to almost non-hygroscopic feature, high strength of extension and good corrosion resistance, polypropylene fiber is almost not eroded by strong acid and strong alkali, but the maximum service temperature should not exceed $100^{\circ} \mathrm{C}[5]$. The filter made from this kind of fiber has a smooth surface and such fiber is also the material most commonly used in all the primary filter. In recent years, thanks to the improvement of environmental protection concept, the various countries all over the world gradually apply polypropylene (PP) fiber to filter, and its superior resistance to strong acid and strong alkali and temperature resistance also gradually improve so as to gradually replace other filters. 
Table 1. Yarn diameter: 0.25mm (400 denier) fabric specifications:

\begin{tabular}{lll}
\hline Warp density (piece/inch) & Weft density (piece/inch) & Weaving width (inch) \\
\hline 42 & 36 & $\geqq 65$ \\
\hline
\end{tabular}

\section{Motivation}

The disposable filter material may lead to waste of energy, and the pollutant of filter material thrown is also the common external cost of all the people. It is hoped to develop green and energysaving products to improve these problems. Currently, the most primary problem is resource exhaustion, in hope of developing the green, environmentally friendly and water-washable filter material products which can be used repeatedly in the shortest possible time. In fact, filter material is used by everyone on a daily basis, and both the filter screen for cooling air and water filter must use filter materials. People become increasingly particular about green and environmental protection and health care, thus it is believed that to some extent its demand will also increase.

Air filtration accounts for about one third in the market of filtration and filter and it is portion with the fastest growth in the filtration field, which has been widely used in industrial field and families.[5] Generally speaking, air filtration is mainly to eliminate the suspended solid material in the air (aerosol), and the diameter of these particles ranges from 0.002 um to $100 \mathrm{um}$; the too small aerosol particle is like gas molecule, while too large particle is easy to be eliminated in the air due to its gravitational settling.[6]

\section{Methodology}

This research conducts the experimental evaluation based on filtration efficiency:

1. Basis and method: Weight Trap of ASHRAE52.1-1992.

2. Experimental instrument: differential pressure gauge is Omega PX654-0.1D5V, particle counter is Las-x CRT type (flow velocity is $2.0 \mathrm{~cm} / \mathrm{sec}$, air volume is $500 \mathrm{CFM}$ ), feeder

3. Specimen preparation: standard dust (50g), standard dust filter screen, filter screen to be measured

4. Standard dust (50g): standard dust

The dust contents stipulated by ASHRAE 52.1 are applied in the course of dust load test; the dust constituents of ASHRAE (weight: \%): 72\% (the dust on the road of Arizona) 2. carbon black: 23\% (particle size $0.08 \mu \mathrm{m}) 3$. cotton velvet: $5 \%(15 \mathrm{~mm} * 0.1 \mathrm{~mm})$.

5. Specimen specifications: the area for standard filter screen and filter screen to be measured is respectively 23.5 inches * 23.5 inches.

6. Test method: first of all, the filter screen to be measured is placed in the filtration modules to measure its initial loss of pressure, and then $50 \mathrm{~g}$ standard dust is measured by the electronic scale; after the filtration modules are started, most dusts are captured by the filter screen to be measured and the rest are captured by standard filter screen; after the standard dust in the feeder is used up, it is weighed after the removal of standard filter screen to calculate the filtration efficiency of filter screen to be measured according to the following formula: the rate of dust collection of air filter collecting efficiency, weight method (above $1 \mu \mathrm{m}$ ), and this method is also called as ATI weight 
method; in this method, the formula is obtained by testing specimen before and after the filter to weigh and compare them.

Dust collection capacity theory is used to show the defined standard of use, and the test for general air cleaning unit is conducted accordingly. Filtration performance is based on the initial performance of new cleaning mesh. When the performance changes due to dust accumulation in the device, it is also called load performance.
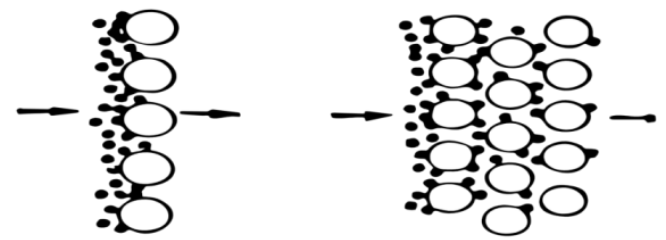

Figure 1 Instruction for filtration method: surface filtration (left figure) and deep filtration (right figure)

The separation mechanism of mechanical filter is shown in Figure 1, which is divided into surface filtration (left figure) and deep filtration (right figure). In terms of surface filtration, the particles in the particle layer accumulated on the surface of filter interfere with each other, so that the separation effect can improve the particle layer sharply produced on the surface of filter to improve the rate of dust collection, meanwhile, the impedance is also increased. In terms of deep filtration, the particles in the spongy or rock-bottom filter are scattered and then separated. Due to the increase in load impedance, filtration efficiency will also decrease. This kind of structure is often used in filtration device and has the large filtration capacity. The filter used for air conditioner generally takes small concentration of air dust as subjects, and in addition to its easy cleaning, due to emphasis on air volume, the increase and decrease of impedance (large dust collection capacity) can make the inner filtration type become ideal. In the earlier period, it is planned to adopt single layer of honeycomb weave, and it is planned to adopt 7 16 layers of 3D fabric to increase the efficiency of deep filtration.

The research direction is the green, energy-saving, long-acting and water-washable filter product, and with the better foundation, it begins to develop towards the direction of other filter material industries (water, gas, etc.) based on the foundation.

During the exhibition, its effect of environmental protection and energy saving will be especially stressed to achieve the goal of green marketing.[7] In terms of prices, the model of small profits but quick turnover is adopted, and the main purpose is to hope that everyone can put more emphasis on environmental protection, thus we can do our bit for the earth and environment through median price. In terms channel, the main promotion method is network marketing; in particular, group purchase and other activities are very popular in the networked society, it is also expected to achieve the effect of publicity through Yahoo Shopping, momo shopping, etc.; besides, the online shopping activities are also combined to achieve the promotional and spreading effect. 
1. Quantified Benefits

Table 2. Quantified benefits

\begin{tabular}{|c|c|c|}
\hline Increased output value $\underline{10000}$ NTD & $\begin{array}{c}\text { New product or service produced } \\
\underline{5} \text { items in total }\end{array}$ & $\begin{array}{l}\text { Derived commodity or } \\
\text { service } \underline{5} \text { items in total }\end{array}$ \\
\hline $\begin{array}{c}\text { Invested research expenditure } \\
2000 \text { NTD }\end{array}$ & $\begin{array}{c}\text { Promoted investment amount } \\
\underline{10000} \text { NTD }\end{array}$ & $\begin{array}{l}\text { Decreased cost } \\
2000 \text { NTD }\end{array}$ \\
\hline $\begin{array}{l}\text { Increased the number of employed } \\
\text { people } \underline{100 \text { persons }}\end{array}$ & Establish $\underline{1}$ new company & Invent $\underline{1}$ piece of patent \\
\hline $\begin{array}{c}\text { A total of } \underline{2} \text { pieces of new or new type } \\
\text { of patents }\end{array}$ & A total of $\underline{2}$ journal papers & $\begin{array}{c}\text { A total of } \underline{1} \text { conference } \\
\text { paper }\end{array}$ \\
\hline
\end{tabular}

2. Non-quantitative benefits

The physical conditions of fibers should be effectively used to produce environmentally friendly, non-polluting and green materials with higher value, higher strength and higher crystallinity to improve the problem; the safety in use may be the most important. As a professional, this is also our first step to produce the product, and the safe materials are used so that the load on the earth is not increased any more.

Although the research and development of green products may cause loss to manufacturers, its following effects may be more important to the society, and manufacturers can make use of the saved resources to improve their products and service, meanwhile they also do their bit for the earth and environmental protection, thus the next generation will have a cleaner and more comfortable place to live in. As a result, society may experience the great evolution, and then the cleaning industry will emerge to promote the generation of more small and micro enterprises, inspire the originality of society and equalize the wealth, and meanwhile the unemployment rate can be indirectly decreased.

3. Effect on overall environment after the completion of this research

(1) Effect on company

The horizontal ties are carried out through plan and academic unit, and the cooperation of industry, official and university is expected to improve the kinetic energy of Taiwan's academic research and introduce new ideas to research fields; the filter products which combine the green and environmentally friendly concepts can achieve the effects of reducing the energy consumption, increase durability and reduce environmental pollution and damage, and the special and highquality products can be developed continuously to increase market share.

(2)The benefits of creation, added value or circulation owned by industry and industrial technology

The company can develop forward-looking products through this plan to further bring positive competition for the improvement of industry and industrial technology. The saved resources can be 
invested into other products and service, and even the rested capitals can be invested into society to do their bit for the earth and country. The most special fact is that the cleaning industry will emerge at the right moment. Due to its lower technology threshold, more people can also be brought into workplace to reduce unemployment rate and change the redistribution of wealth.

\section{Conclusion}

The research results show that the horizontal ties are carried out through plan and academic unit, and the cooperation of industry, official and university is expected to improve the kinetic energy of Taiwan's academic research and introduce new ideas to research fields; the filter products which combine the green and environmentally friendly concepts can achieve the effects of reducing the energy consumption, increase durability and reduce environmental pollution and damage, and the special and high-quality products can be developed continuously to increase market share. In addition, the benefits of creation, added value or circulation owned by industrial technology can develop forward-looking products for the company through this plan to further bring positive competition for the improvement of industry and industrial technology. The saved resources can be invested into other products and service, and even the rested capitals can be invested into society to do their bit for the earth and country. The most special fact is that the cleaning industry will emerge at the right moment. Due to its lower technology threshold, more people can also be brought into workplace to reduce unemployment rate and change the redistribution of wealth.

\section{References}

[1] Lin, S.T., Chen, T.J., Lin, K.F., and Wang, S.P. (2012) Research into the Manufacturing and R\&D Management of New Optical Grating Cultivation Net, 2012 International Academic Seminar for the Business Incubation of Micro-enterprises

[2] Lin, S.T., Wang, S.P. Chen, T.J., and Lin, K.F. (2013) Discussion on the Optimized Manufacturing Parameter of New Optical Grating Cultivation Net, International Academic Seminar for the Innovation of Middle and Small-sized Enterprises in Asia-Pacific

[3] Chi, Y.H. (2015). Research into the Indoor Air Quality and Acceptability of Innovative Products - a Case Study of Catalytic Filter Screen Company, master's thesis, EMBA, school of Management Development, Feng Chia University.

[4] Lin, S.T., Chen, T.J., Lin, S.J., and Lin, K.F. (2014) Research into the Application of New Optical Grating Cultivation Net to the Optimization of Rice (Penglai Rice) Planting Parameter, International Seminar for the Regional Economy in Asia and Local Industrial Development

[5] Lin, S.T., Lin, S.J., Lu, C.Y., and Lin, K.F. (2015) Research into the Management of

Agricultural Pest and Disease Damage for the Environmental Control in New Optical Grating Mesh, International Seminar for the Regional Economy in Asia and Local Industrial Development

[6] Robert Mcilvaine, Mcilvaine Company, Northfields, IL 60093 USA

[7] Peng, T.K and Hsieh, Y.L. (2008). Change of Taiwan's Agricultural Structure and Priority of Agricultural Policy. Journal of the Agricultural Association of Taiwan, 9(6). 